\title{
Integrated, automated and robotic process for building upgrading with prefabricated modules
}

\author{
Kepa Iturralde $^{\mathrm{a}}$ and Thomas Bock ${ }^{\mathrm{a}}$ \\ ${ }^{a}$ Chair of Building Realization and Robotics, Technical University of Munich, Germany \\ E-mail: kepa.iturralde@br2.ar.tum.de, thomas.bock@br2.ar.tum.de
}

\begin{abstract}
-
Adding manually thermal insulation onto an existing building is an inefficient and labor-intensive process. To avoid such manned labor on site, recent approaches have been developed for installing prefabricated modules onto existing buildings' envelopes. However, the monitoring and the analysis of these approaches expose that these procedures are still time-consuming and inaccurate. In mass manufacturing industries, robots facilitate time reduction while ensuring accuracy. Nevertheless, the main challenge for adopting robotized manufacturing and installation processes in building renovation relies on the constant customization of the prefabricated modules, both off-site in the factory and on-site in construction. In pursuance of improving such situation, the authors conceived a novel process that automates the customization, the manufacturing and the installation processes of the modules. The solution integrates and links systems such as accurate coordinate acquisition, parametric design, digital manufacturing of elements and robotic assembly and installation of the modules. The main body of this paper describes the structure of this novel conceptual process. This process has been initially proofed, addressing mainly the feasibility and the correct sequence of each of the steps. The results show that this novel process, after further development, can be a solution for gaining efficiency on the building renovation processes.
\end{abstract}

Keywords -

Renovation, robotic, parametric, prefabrication

\section{Introduction}

Achieving a zero-energy consumer building stock is a goal of the European Union [1]. Currently, the most used and "traditional" method comprises the addition of an insulating layer on the exterior wall or facade of the building. After that, the operators spread mortar or place a waterproof material to protect this insulating layer. This procedure is time consuming and requires scaffold installation in front of the building's façades [2]. In order to gain efficiency of the process, the European Union prepared a research call [3] defining the next main requirements for the applicant proposals:

- Use prefabricated modules with integrated energy efficiency devices and renewable energy sources.

- Use advanced computer based tools for integrating the value chain over the life cycle of the project.

- Move from individual manufacturing to mass-customization.

- Reduce the installation time by at least $30 \%$, compared to a typical renovation process for the building type. For that purpose, high accuracy is needed

The BERTIM project (2015-2019) [4] was selected within this call. The main challenge relies on using timber-based prefabricated modules to cover existing buildings' envelopes. The results of a preliminary demonstrator [5] of this research project showed that improvements in manufacturing and installation were still required in order to obtain a more efficient process.

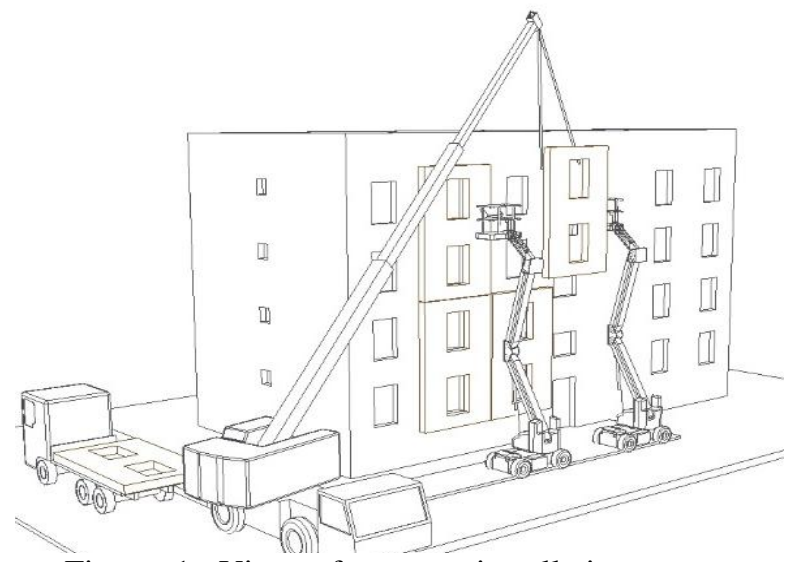

Figure 1. View of current installation process techniques of prefabricated modules for building refurbishment with mobile cranes.

The remainder of this paper is to explain and verify an integrated process that ameliorates the procedures of the 
demonstrator of the project [5]. For that purpose, some points have been outlined. First, the main challenges of the research are presented in chapter 2. After that, a solution that spans the research gap is defined in chapter 3. The verification process is described in chapter 4 . Finally, in the last chapters, the future necessary works are outlined.

\section{Multi-Aspect research gap}

According to previous studies [5], the procedures used for adding a 2D module onto existing buildings are still excessively time-consuming. Time consumption affects different aspects: the design or layout-planning period, and the manufacturing and installation processes This yields an increase of cost and therefore prefabricated solutions lose competitiveness in comparison to manual procedures [2]. As outlined in previous papers [5], it affects especially to the on-site installation phase. In other industries, a solution to these problems is the use of robotic and automated technology in order to improve the performance. There have been advances in product flexibility and flexible automation [6]. But normally, robotic manufacturing requires a standardized product. Moreover, building renovation requires fully bespoke products, adjusted to the existing geometry and function. The product's size shape changes constantly. Therefore, a flexible robotic manufacturing process is needed for a customization. There are already some experiences where the linkage between a parametric design and a robotic control was developed [7],[8],[9]. These studies are focused on complex geometries more than the assembly of fully prefabricate units.

Besides, preliminary research [5] determine that the existing manufacturing processes produce $2 \mathrm{D}$ timber modules with excessive tolerances, with around 10+/$\mathrm{mm}$ of deviations. For a highly prefabricated module in the rest of the construction industry, i.e. curtain wall modules, the tolerances cannot exceed $1+/-\mathrm{mm}$. In previous studies, it was determined that the accuracy of the modules and the placement of the connector was crucial for a proper fasten. Furthermore, in the BERTIM project, it has been concluded that the insertion of services, and therefore, pipes and ducts must fit. This requires high accuracy.

Between the 2D module and the existing building, there is a connector that consists in two parts. This was further explained in previous publications [10]. One part, (Part 1) is fixed onto the existing building and the other part (Part 2) is fixed onto the 2D module. This way, the 2D module is supported on the connector, which in turn is supported on the existing building. With current techniques, accurately locating Part 1 of the connector is time consuming. Prior to this research, an interfacing
Matching-Kit ${ }^{1}$ has been defined and proved, which corrects the possible deviations of Part 1. The problem arises with the placement of Part 2 onto the 2D module; the location differs from case to case.

\subsection{Information workflow, data acquisition and layout-planning,}

In current processes, there is a lack of a continuous information workflow. This situation leads to errors and misunderstandings. The potential benefits of an integrated process were highlighted before [11] and considered BIM as a starting point for integrating the whole process. But in this case, the link between the robotic tasks and BIM needed further development.

Normally the process of building renovation with prefabricated modules starts with the existing building's data acquisition. Progress has been made to implement 3D laser scanning for acquiring the data of spatial environment [12]. The question arises, if this technique is accurate enough for manufacturing and installing parts onto a previously built environment with high enough accuracy. Besides, the point cloud management might gather too much information to handle, at least for the façade renovation. Moreover, a faster and more efficient procedure for building renovation is to prioritize key points. This is based on the selection of only necessary information and the discrimination of the unnecessary geometrical information. This technique is already developed [13]. At this point, the problem relies on the location of the connector; it is also necessary to determine the location of the connector, which might be placed in a non-expected or undesired location. If the location of this connector is not measured accurately, or it is not placed accurately, the $2 \mathrm{D}$ module does not fit [10].

Finally, the re-design or layout-planning of the 2D modules needs to be used by a dedicated parametric software. Software such as Dietrich's and RenoBIM [4] are developed (or are being developed) for a fast adaptation of the modules' geometries. Recent studies suggest that the software information can be integrated [14] in the manufacturing and the installation processes. Addressing this, it is necessary to define a specific programming system that integrates the process.

\subsection{Off-site manufacturing phase}

The manufacturing accuracy of single elements can be gathered currently with $\mathrm{CNC}$ machines and even with robots. However, the automation level is limited on the following assembling phases. Currently, the assembly process is a purely manual procedure. Besides, there is a growing complexity of the $2 \mathrm{D}$ modules. This

\footnotetext{
${ }^{1}$ Publication under review process
} 
means that more and more components and elements are included during the assembly process. This negatively affects the sought automation of the manufacturing system.

\subsection{On-site installation phase}

With current techniques, excessive re-work is necessary after the modules are placed and fixed onto the existing building's façade [5]. The prefabrication degree of the $2 \mathrm{D}$ modules is, depending on the case, around $50 \%-70 \%$. This means that the rest of the tasks need to be finished on-site.

In any manner, there is no dedicated machinery for installation processes. Currently, the cranes and scaffolding systems are not suitable to this specific purpose. In previous research [15][16] there has been a study of different options for a robotic installation of modules. It must be mentioned that experiences such as the Hephaestus project [17] are relevant to carry out the installation process of the $2 \mathrm{D}$ modules efficiently.

\subsection{Objective}

For reducing the overall time, the main objective consists on integrating all phases, from existing building's data acquisition to robotic 2D module installation. It is necessary that, from the acquisition of the key point coordinates of the existing building, the information for manufacturing and installing processes is linked. The solution should enable high prefabrication degree and accurate production and installation of modules. For that purpose, robotizing the off-site assembly and the on-site installation process of the $2 \mathrm{D}$ modules is desirable.

Since the 2D modules are customized products, in principle, the robot path and grasping for the assembly process should be programmed specifically for each $2 \mathrm{D}$ module. Importantly, there is one point to take into account. The 2D modules follow a pattern, they have many common characteristics and their layout changes according to some parameters. Therefore, the robotic programming could respond also to that parametric characteristic, and subsequently, if the controlling algorithms were arranged adequately, it would not need to be adjusted specifically for each 2D module.

\section{Integrated process}

In order to address the gap stated before, a solution was drafted. The proposed solution relies on a parametric software based on a visual programming language (VPL). This VPL functions as an integrator of several main points: the data acquisition of the existing building, the 2D module design/layout planning, the robotic manufacturing, and the robotic installation phases.
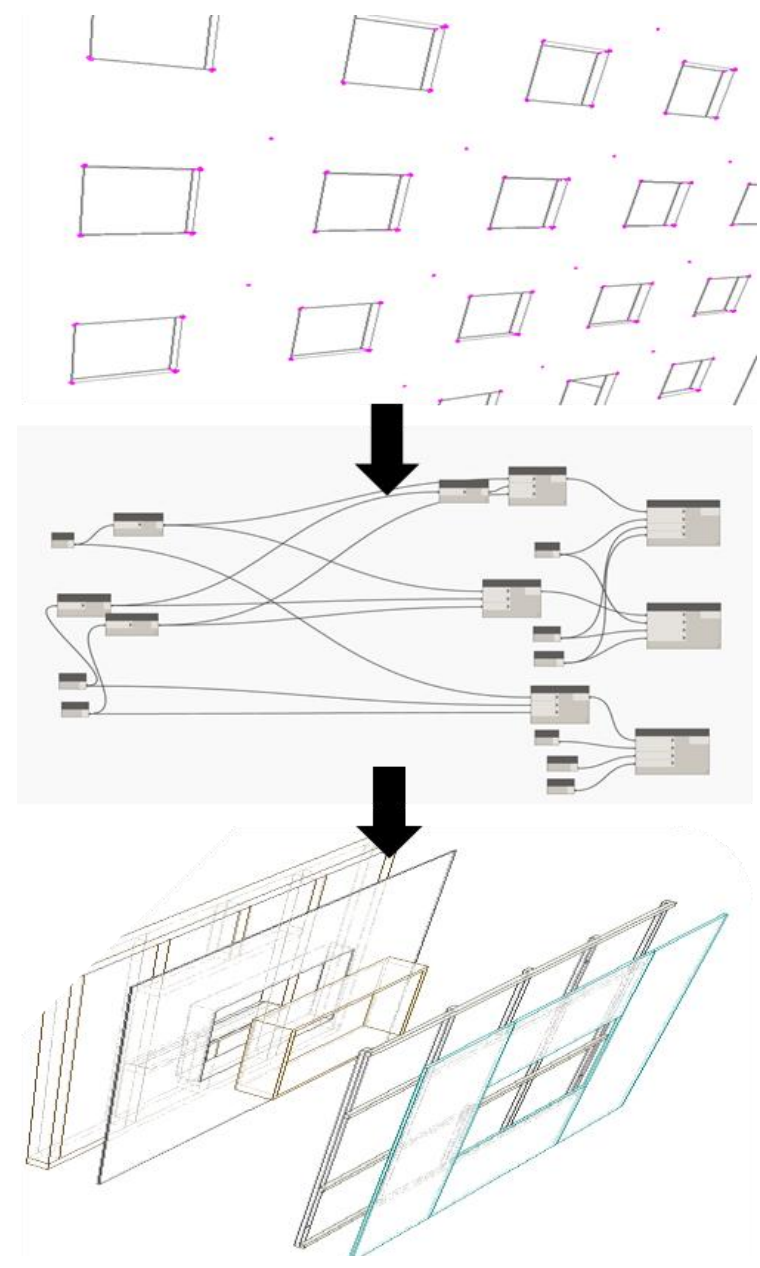

Figure 2. Necessary points' coordinates in a façade for being inserted in the VPL and definition of every element of the $2 \mathrm{D}$ module by using the VPL.

As a primary input of the solution, only the twelve key points of the existing building would be necessary for defining each of the 2D modules. Four points would refer to the perimeter corners of the $2 \mathrm{D}$ module, and the other eight would be correlate to the window and its sill. In the case of a regular façade, that would mean that a grid similar to Figure 2. For now, the scope of the solution considers only very simple facades without terraces and balconies. As an output, the VPL would produce the necessary scripts for guiding the path and grasp-planning of the robots. The robotic environment used in this novel integrated process relies on Robotic Operating System (ROS) [17] and implies both off-site manufacturing processes and on-site installation processes. 


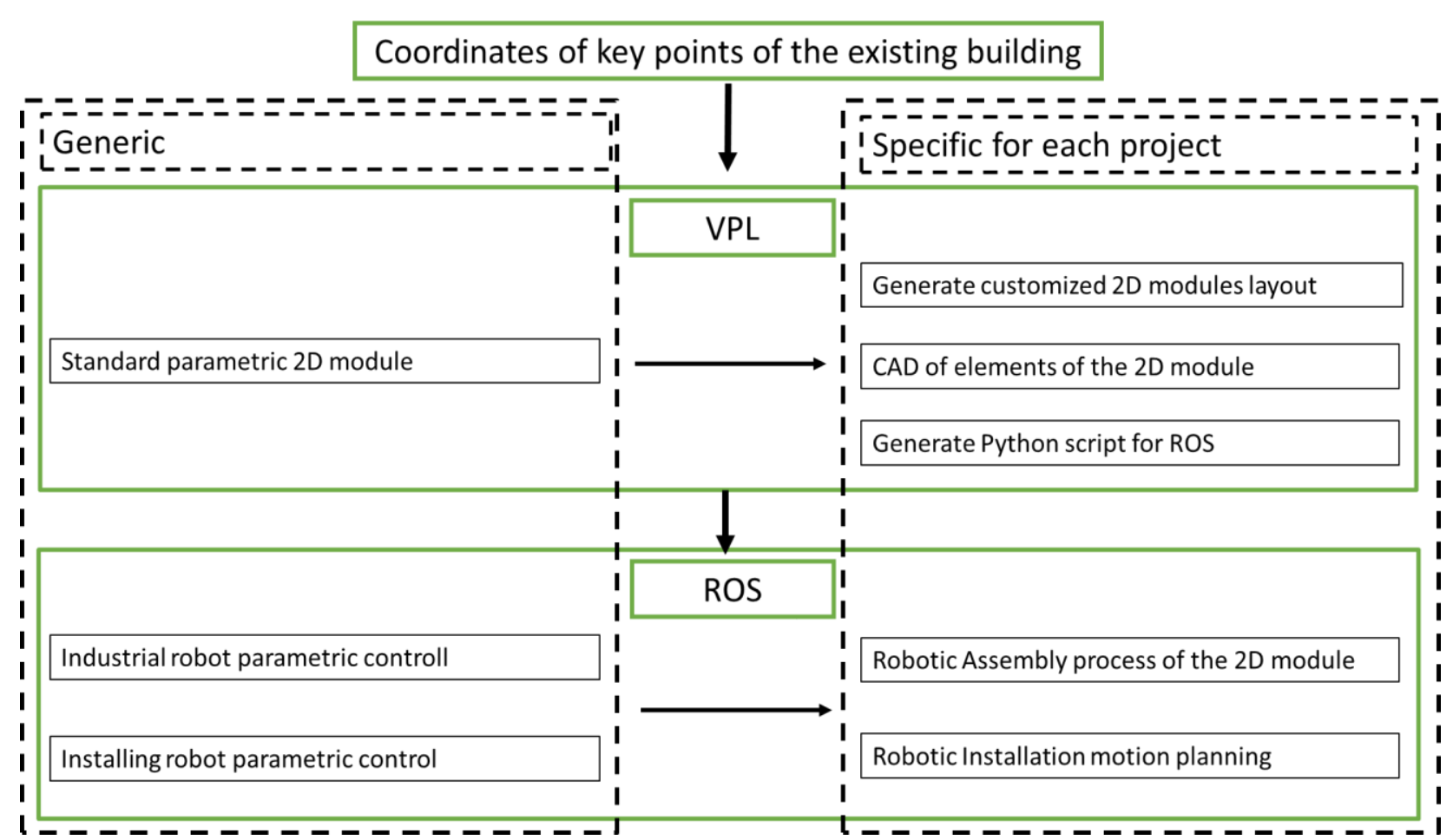

Figure 3. Scheme for the integration of the process.

\subsection{Setting out of the standard 2D module}

The term "standard" means that there is a library of solutions for the $2 \mathrm{D}$ module, with parametric variables regarding the size, shape and outer finishing material. In the case explained in this paper, the 2D module was defined to cover only flat facades, with no terraces or balconies, only with flat windows. The design of the 2D module was based on different layers. The inner layer of the wall was a flexible insulating material that adapts to the irregularities of the existing wall. Then it was followed by a flexible moisture barrier layer. This was to prevent the humidity of the interior of the building from damaging the $2 \mathrm{D}$ module itself. The main part of the $2 \mathrm{D}$ module was a timber frame, which was filled with insulation. After that a board was placed for rigidizing the timber frame. Following the criteria of previous research, the services were allocated into this timber frame, with extra insulation on the outer surface of the timber frame. For protecting the module from outdoor weather, a waterproof layer fully covered the outer surface. With current techniques, the overlapping of this layers is achieved manually on the site and after that, a finishing layer is placed and windows are inserted in the voids of the timber frame. But the layout and design of this research defined a prefabrication degree of $100 \%$, including the services for RES. The studs and the battens have a maximum separation of 600 $\mathrm{mm}$.

\subsection{Setting out of the robots motion and grasp planning}

In this integrated process, two types of robotic systems were conceived. On the one hand, there were the already commercialized industrial robots for the assembly of the 2D modules on the factory. On the other hand, there were the installation robots on the construction site.

In the off-site structured environment in the factory, the robot and the automated manufacturing machines and the 2D module's elements are in recognizable positions. Therefore, an open-loop control system without sensory feedback is foreseen. The visual programming node generates a sequence of the assembly process for each of the elements and the 2D modules. For the assembly process off-site, as defined already before [11], the VPL generates also different types of files such as CAD or STL for the CNC machines and robots.

Besides, on the site, the situation is different. This is an unstructured environment, where the robot needs a calibration and guidance. In first considerations, the closed-loop control system was chosen as the best situation, since it is presumed the robotic system will need a feedback of its location. For that purpose, the 
selected key points of the building (the points that were acquired during the data acquisition) can be used as a reference for calibrating the robot. There are currently no marketed robots for the full installation of $2 \mathrm{D}$ modules. Several options have been envisaged [15],[16],[17]. As a first approach in this research, a stacker crane or Cartesian robot was defined. This Cartesian robot would host a Modular End-Effector similar to the one defined by the authors [19]. This system resembles the already commercialized systems for Automated Storage and retrieval Systems [20].

\subsection{Proposed sequence}

A building renovation project with prefabricated products requires parallel processes. On-site and off-site tasks need to be synchronized. The integrated on-site process sequence is defined with the next points:

1. The process starts with preliminary data collection of the wall-façade. Basically, it consists of the measurement of the façade's key points by a digital total station. If the façade is planar, without balconies, the task can be achieved from the ground of the building. The key points are located in the window sill and perimeter of the façade.

2. These coordinates are listed and inserted in the VPL to recreate the façade and the layout of the modules. This step is not attached to the site nor the off-site factory, so it can be carried out in any location.

3. The installation of the robot body. For this research, the robot would be based on a vertical stacker crane. This robot must be calibrated properly according to the $0,0,0$ or origin point selected within the building.

4. At this point ${ }^{2}$, the onboard tools place the connector's Part 1 with an accuracyof 20 +/-mm.

5. The onboard measuring device detects the location of Part 1.

6. The Matching-Kit (MK) is fixed by the robot and a planar, known situation is generated.

7. The 2D module is fixed onto Part 1 and the MK. In parallel, the $2 \mathrm{D}$ modules are manufactured using the generated information by the VPL.

a) The data is introduced into the variables of the VPL. Automatically, the layouts of the 2D modules are generated.

b) The CAD and/or STL files are exported by the VPL for manufacturing the parts of the $2 \mathrm{D}$ modules. The generation of a CAD file out of parameters is not a novelty; it is used by other processes [11].

c) Every single part is machined and routed in a CNC machine. This way it can be guaranteed that the parts are contoured and milled accurately.

\footnotetext{
2 Points 4, 5 and 6 are described more extensively in another publication under review process.
}

d) The Matching-Kits are produced as an interface. This MK can be produced on-site or off-site.

e) Finally, the assembly of the parts of the 2D modules is performed by the robotic tools. The scripts from the VPL defines the trajectory planning of the desired task, as well as the manipulator control system.

As stated before (ISARC 2016), the process sequence is modifiable depending on some concepts. The arrangement of the on-site sequence $(1,2,3,4 \ldots)$ and the off-site sequence (a, b, c, d...) steps presented in this chapter are variable. For instance, if the placement of Part 1 is performed accurately (step 4), the MKs (step c) would not be needed.

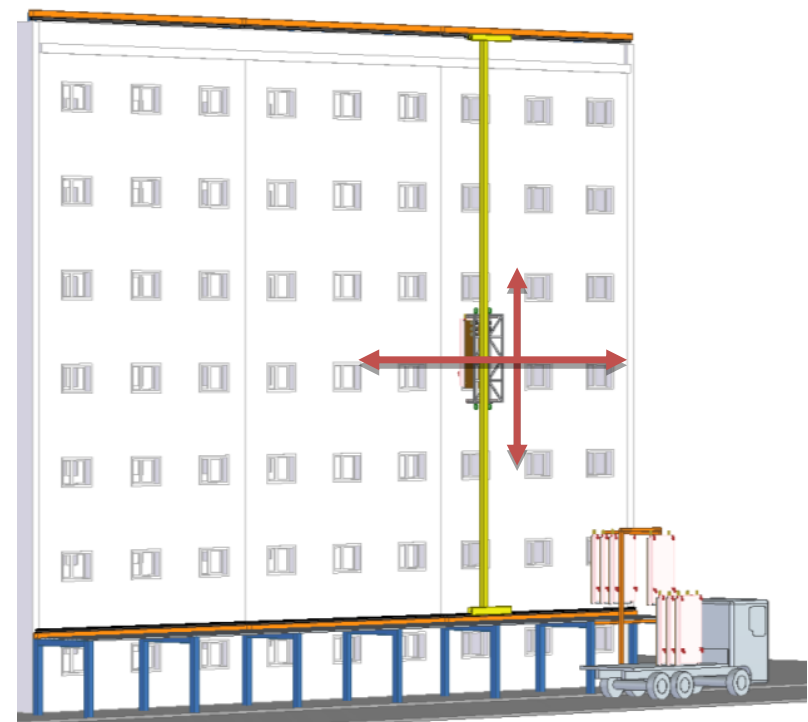

Figure 4. Installation process by a stacker crane (also known as vertical bridge crane).

\section{Verification of the integrated process}

For the verification of the process, some tests were achieved. The tests consisted of a laboratory experiment and a virtual simulation. The main objective of the tests was to verify the correct sequence of the process.. The verification was achieved in two main scenarios: (1) the robotic assembly of the $2 \mathrm{D}$ modules off site with a real robot, and (2) the installation phase on site, virtually. The VPL, Dynamo(C [21], was used as an integrator. Dynamo $($ is a Visual Programming system developed by Autodesk used mainly for Design of parametric objects.

For this test, a sample of a 2D module was used. This sample was a scaled and simplified mock-up of a 2D module, based on timber frame. The 2D module was defined in Dynamo $(C)$ environment and was parameterized depending of the 4 points of the perimeter of the 2D module. The module was based on eight different timber elements and four different 
connectors. For the test, the 2D modules did not have any window, and therefore the eight points referred to in the previous chapter were not necessary. In a previous publication $^{3}$, it is explained how the key points of the location of Part 1 were measured.

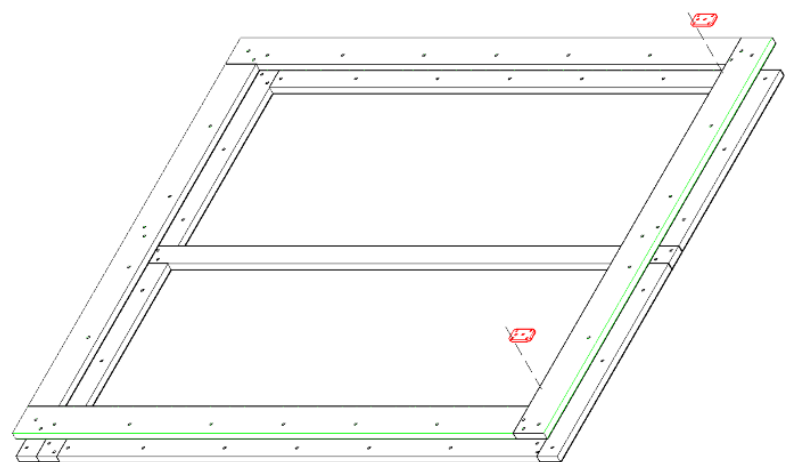

Figure 5. Definition of the 2D module for the Test.

The module was dependent to the existing wall's key points. During the customization stage in Dynamo $\subset$, the data from the coordinates were listed in data sheet file. From here, the sorting node of Dynamo $@$ first generated a solid that defined the façade or the wall, in the case of the test. The STL files generated by Dynamo $\odot$ were used by a 3D printer for generating the the Matching-Kit interfaces. Finally the CAD model.dwg file of the timber-frame element are used for routing and milling each of the elements.

Once the elements were accurately produced, the assembly process started. For the controlling system of the robots, ROS [17] was used. ROS comprehends and integrates a set of robot software frameworks. In the case of this research, ROS was used for the industrial robots in the laboratory, in addition to the installation robots in a virtual simulation.

\subsection{Off-site robotic assembly of the 2D modules}

For this test, the lightweight Jaco $@$ robot by Kinova was used. The Jaco( robot can be installed in ROS environment. Prior to the performance of the robot, the robotic workstation was structured according to the base $0,0,0$ origin coordinate. The parts of the modules were also placed in a known location according to this point. The task consisted on moving these elements to a pose goal within the 2D module, according to the 2D layout. The Python script generated in Dynamo $@$ was taken by the Gazebo-Moveit application within ROS environment. The script sequenced the assembly of each of the elements, from the first element to the eighth

\footnotetext{
${ }^{3}$ Publication under review process
}

element. An advantage was that the operator did not need to check the location of the elements, since the task was given directly by the robot.

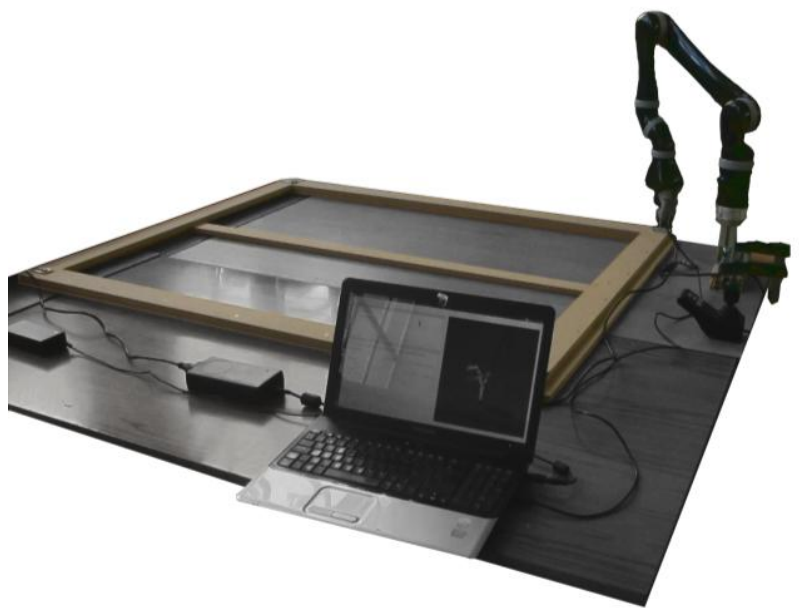

Figure 6. The Jaco@ robot operating with MoveIt ROS.

On this test, only the pick and place task of a relatively small and lightweight element was achieved. For this test, the screwing process was carried out manually. To achieve this robotically, another robot with higher payload and torque capacities would be necessary.

\subsection{On-site robotic installation of the 2D modules}

This first verification of the installation phase has been achieved virtually, since the robotic stacker crane is not operative for buildings and outdoor conditions. An URDF file that replicates the stacker crane was generated. This URDF was introduced to the ROSGazebo environment. Then, the stacker crane robot performed a task that simulated a simple drilling operation according to the given poses by the script.
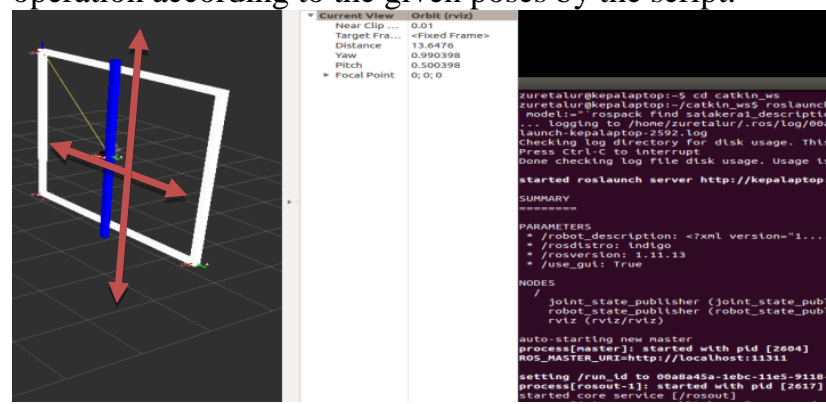

Figure 7. The stacker crane or Cartesian robot in Gazebo ROS.

For now, only the task of the drilling process has been tested and the results are satisfactory, but this process needs to be further developed. 


\section{Future work}

The results of the laboratory verification suggest future work considerations. For the assembly process, the some issues have been foreseen. For instance, the robotic assembly would require sequencing coordinately with the on-site works. The start of 2D module manufacturing depends on the size of the building, the bigger the building is, the earlier the manufacturing process needs to start. Related to that, the authors have envisaged two sequences within the manufacturing process of highly accurate $2 \mathrm{D}$ modules. The first is based on the prior routing of every single part of the module and the assembly of them. This is the process that has been explained in the paper. On the contrary, the process can be inverse, by assembling the elements first and then complete final routing of the entire 2D module. In other words, the 2D module can be produced with high tolerances, and then inserted and routed in a CNC machine to get the required contour with precision. Besides, regarding the assembly process, The center of gravity of each of the 2D module's element should be calculated and taken into consideration before inserting the data on Moveit and Graspeit applications. This way, it should be possible for the robot to grasp the elements in the right position, close to the respective center of gravity. In this research collision detection hasn't been approached and definitely it is an aspect to take into consideration. The unstructured workplace in the industrial settings must be considered, so the assembled elements are disposed in an aleatory manner.

For the installation process, some other issues must be taken into account. If the stacker crane (or Cartesian robot) system is used, it will depend on the accuracy of the carrier and the linear and rotary encoders. If these devices are not precise enough, another device or sensor would be necessary for positioning. Based on the previous point, a repeatability of the pose will need to be tested. The stacker crane can be up to 30 meters high. At this height, the carrier suffers some balancing and therefore, counter measures will be necessary, such as suction cups for holding the end-effectors.

Finally, this very first approach is limited to simple facades, thus it does not include facades with balconies or terraces. In the future, standard libraries for covering such building parts will be necessary. Depending on the complexity and the size of the building to be renovated, the robots' controlling system might not be able to handle the data, therefore, splitting the renovation project might be necessary.

\section{Conclusions}

The existing techniques for manufacturing and installing prefabricated modules for upgrading building facades needs to be raised to a more efficient and automated method. The integrated process presented in this paper offers possibilities for reducing inefficiencies and increasing accuracy on the building renovation process. The proposed process or sequence, based on VPL and robotic assembly and installation, has been verified in a laboratory environment with satisfactory results.

The 2D modules are in general very similar, but they have many particularities; size and location of elements. These peculiarities are demanded by the geometry of the existing building. This means that the robots, both in the industrial settings and on the construction site, need to perform similar operations, but depend on specific parameters each time.

During the verification, with only the given input of the existing building's coordinates, the VPL processed the data for the assembly robot that performed the assembly of the 2D module. Furthermore, in a simulation achieved in ROS environment, it has been tested that 2D module installation robots have a big potential. Both assembly and installation robotics still need more improvement. In the future, each step of the proposed sequence can be further developed and tested separately.

\section{Acknowledgments}

This paper has been drafted thanks to the funding from the European Union's Horizon 2020 research and innovation program under grant agreement No 636984.

\section{References}

[1] European Commission. A Roadmap for moving to a competitive low carbon economy in 2050, European Commission, Brussels, 2011.

[2] BKI. Baukosten Gebäude Altbau, Statistische Kostenkennwete, BKI GmbH, Stuttgart, Germany, 2014.

[3] European Commission. Manufacturing of prefabricated modules for renovation of building. On-line:

http://ec.europa.eu/research/participants/portal/des ktop/en/opportunities/h2020/topics/ee-012014.html, Accessed: 30/01/2018

[4] BERTIM consortium. Building energy renovation through timber prefabricated modules. On-line: http://bertim.eu, Accessed: 30/01/2018

[5] Iturralde, K., Linner, T., Bock, T. First Monitoring and Analysis of the Manufacturing and Installation Process of Timber Based 2D Modules for Accomplishing a Future Robotic Building Envelope Upgrading. In Proceedings of ISARC 2017, pages 65-73, Taipei, Taiwan, 2017.

[6] Thomas, U., Stouraitis, T., and Roa, M. A. 
Flexible assembly through integrated assembly sequence planning and grasp planning. In Automation Science and Engineering (CASE), IEEE International Conference, pages 586-592, Gothenburg, Sweden, 2015.

[7] Braumann, J., Brell-Çokcan, S., Parametric Robot Control: Integrated CAD/CAM for Architectural Design. In Proceedings of the 31st annual conference of the Association for Computer Aided Design in Architecture, pages 242-251, Calgary, Canada, 2011.

[8] Lloret, E., Shahab, A.R., Mettler, L., Flatt, R. J., Gramazio, F., Kohler, M., and Langenberg, S. Complex concrete structures: Merging existing casting techniques with digital fabrication. Computer-Aided Design 60:40-49, 2015

[9] King, N., Bechthold, M., Kane, A. and Michalatos, P. Robotic tile placement: Tools, techniques and feasibility. Automation in Construction, 39:161166.

[10] Iturralde, K., Linner, T., Bock, T. Development of a modular and integrated product-manufacturinginstallation system kit for the automation of the refurbishment process in the research project BERTIM. In Proceedings of ISARC 2016, pages 1081-1089, Auburn, USA, 2016

[11] Meschini, S., Iturralde, K., Linner, T., Bock, T. Novel applications offered by Integration of Robotic Tools in BIM-based Design Workflow for Automation in Construction Processes, In Proceedings of the CIB IAARC W119 Workshop, Munich, Germany, 2016.

[12] Ishida, K., Kano, N. A Study on the Optimization Method for Panel Layout Problem in Drywall. In Proceedings of ISARC 2011, pages 373-374, Seoul, Korea, 2011.

[13] Dietrich's. Bauen im Bestand mit Dietrich's Laseraufmaß.Online:https://www.dietrichs.com/de /anwendungen/bauen-im-bestand-mitlaseraufmass/. Accessed: 30/01/2018

[14] Vareilles, E., Santa, A.B., Falcon, M., Aldanondo, M. and Gaborit, P. Configuration of high performance apartment buildings renovation: a constraint based approach. In Industrial Engineering and Engineering Management (IEEM), 2013 IEEE International Conference. Pages. 684-688.

[15] Iturralde, K., Bock, T. Robotic upgrading of postwar social dwelling envelopes. In Proceedings of ISARC 2013, pages 84-91, Montréal, Canada, 2013.

[16] Iturralde, K, Linner, T., Bock, T. Comparison of Automated and Robotic Support Bodies for Building Facade Upgrading. In Proceedings of ISARC 2015, pages 1-8, Oulu, Finland, 2015.
[17] ROS. On-line: http://www.ros.org/, Accessed: 30/01/2018

[18] HEPHAESTUS consortium. On-line: http://www.hephaestus-project.eu/ , Accessed: 30/01/2018.

[19] Iturralde, K., Linner, T., Bock, T. Development and preliminary Evaluation of a concept for a Modular End-Effector for automated/robotic Facade Panel Installation in Building Renovation. In the Proceedings of 10th Conference on Advanced Building Skins. Bern, Switzerland, 2015.

[20] Kinney, T., Interlake Inc, 1988. Stacker bin shuttle. U.S. Patent 4,756,657

[21] Dynamo. On-line: http://dynamoprimer.com/en/, Accessed: 30/01/2018 